\title{
Targeting FGF for third-line treatment of mRCC
}

Results of the GOLD phase III randomized controlled trial of dovitinib versus sorafenib for metastatic renal cell carcinoma (mRCC) - in the third-line setting-reveal no difference in efficacy between the two drugs. This finding has led to doubts about whether inhibition of fibroblast growth factor (FGF) signalling is an appropriate treatment strategy for patients with refractory mRCC.

Current standard first-line and secondline treatments for mRCC are targeted agents against the vascular endothelial growth factor (VEGF) or mammalian target of rapamycin (mTOR) pathways, but nearly all patients experience progression on second-line therapy. FGF signalling has been implicated in escape from the antiangiogenic effects of VEGF-targeted agents, leading to the idea of incorporating FGF inhibition into third-line therapy. Dovitinib targets the FGF receptor (in addition to the VEGF receptor and platelet-derived growth factor receptor) and has demonstrated tumour reduction in a phase I study of mRCC.
570 patients with mRCC who had previously received one VEGF-targeted therapy and one mTOR inhibitor (in either sequence) were randomized to receive dovitinib $(n=284)$ or sorafenib $(n=286)$ until they experienced disease progression, unacceptable toxicity, withdrew from the study or died.

\section{4 ....median overall survival of 11 months highlights the need for novel agents in this setting 77}

Median progression-free survival (the primary end point) was similar between groups: 3.7 months (95\% CI 3.5-3.9)

for patients who received dovitinib and 3.6 months (95\% CI 3.5-3.7) for those who received sorafenib. Median overall survival was 11.1 months (95\% CI 9.5-13.4) and 11.0 months (95\% CI 8.6-13.5) for the dovitinib and sorafenib arms, respectively.

These disappointing outcomes seem to suggest that targeting FGF is not the right strategy to tackle resistance to
VEGF-targeted drugs. Previous studies have demonstrated elevated levels of FGF2 in the plasma of patients who experienced disease progression while receiving VEGFtargeted therapies, but the current study did not reveal such an increase. However, the investigators note that $92 \%$ of their cohort received a VEGF inihibitor first and an mTOR inihibitor second, making it possible that elevated FGF2 levels could have fallen during second-line treatment.

Despite the failure to demonstrate superiority of dovitinib in this context, the researchers hope their results will provide useful reference data for future studies. If nothing else, the median overall survival of 11 months highlights the need for novel agents in this setting.

Sarah Payton

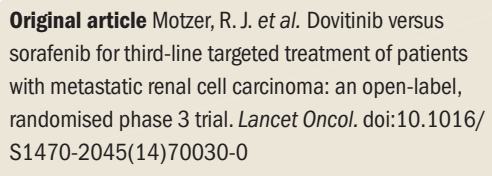

Original article Motzer, R. J. et al. Dovitinib versus sorafenib for third-line targeted treatment of patients with metastatic renal cell carcinoma: an open-label, randomised phase 3 trial. Lancet Oncol. doi:10.1016/ S1470-2045(14)70030-0 